\title{
ANDROESTERILIDAD EN LÍNEAS E HÍBRIDOS DE MAÍZ DE VALLES ALTOS DE MÉXICO ${ }^{1}$
}

\author{
Margarita Tadeo ${ }^{2}$, Alejandro Espinosa ${ }^{3}$, Ana María Solano ${ }^{4}$, Rafael Martínez $^{4}$
}

\section{RESUMEN}

Androesterilidad en líneas e híbridos de maíz de Valles Altos de México. En los años 1995 a 1997 se evaluaron combinaciones de líneas con progenitores androesteriles para encontrar genotipos con capacidad restauradora. Siete líneas restauraron la fertilidad masculina. También se han ubicado algunos híbridos con progenitores con androesterilidad de excelente rendimiento $(12.230 \mathrm{~kg} / \mathrm{ha})$, con posibilidades de uso comercial (UHS95E0123 X P2-1). La capacidad restauradora de la fertilidad masculina (100\%) de las líneas P2-1, IA491, IA49-2, EHT-29-1, EHT-49-3, se confirmó en evaluaciones de progenies, además en 1997 y 1998, se obtuvieron las versiones isogénicas con la tercera y hasta cuarta retrocruza, de las líneas élite de la UNAM, de las líneas hembras de los híbridos PUMAS, en uso comercial como es PUMA 1157 y PUMA 1075, también se cuenta con la versión androesteril de los progenitores del INIFAP denominados $\mathrm{H}-50$ y H-48, recomendados para los Valles Altos, así como algunas líneas del CIMMYT, con lo cual podría favorecerse la producción de semilla de los híbridos que se generan con estos materiales.

\begin{abstract}
\end{abstract}
Andro-sterility of corn lines and hybrids in Valles Altos of Mexico. Lines combinations of andro-sterile progenitors were evaluated to find genotypes with restoring capacity during the years 1995 to 1997 . Seven lines were defined and verified to restore the male fertility. Also, some hybrids with andro-sterile progenitors of excellent yield (12230 kg/ha) and possibilities of commercial use (UHS95E0123 X P2-1) were located. The male fertility restoring capacity (100\%) of the lines P2-1, IA49-1, IA49-2, EHT-291, EHT-49-3 were confirmed in progeny testing. Besides in 1997 and 1998, the isogenic versions were obtained with the third and up to the fourth back-crosses, from the elite lines from UNAM and the PUMAS female hybrids (in commercial use as the PUMA 1157 and PUMA 1075). Also, there is a version of the andro-sterile progenitors from INIFAP, denominated as $\mathrm{H}-50$ and $\mathrm{H}-48$, recommended for the Valles Altos; as well as some lines from CIMMYT, which could favor the seed production of the hybrids that are generated from these materials.

\section{INTRODUCCIÓN}

En la producción de semilla de híbridos de maíz, el despanojamiento oportuno y adecuado es fundamental para obtener la calidad e identidad genética correspon- diente, este proceso implica además costos por uso de jornales, el empleo de la androesterilidad es un mecanismo que puede facilitar la producción de semilla híbrida, por ello en el programa de mejoramiento genético de maíz de la Facultad de Estudios Superiores

1 Presentado en la XLV Reunión Anual del PCCMCA en Guatemala, Guatemala. 1999.

2 Coordinadora de Cátedra de Investigación en Semillas y Profesora de Asignatura, Ingeniería Agrícola, FESC-UNAM, Cuautitlán Izcalli, México. E-mail: tadeorobledo@yahoo.com y tarm@servidor.unam.mx

3 Investigador Titular de Producción y Tecnología de Semillas, INIFAP-SAGARPA. Campo Experimental Valle de México, Km. 18.5 Carretera México - Lechería, Apartado Postal 10, C.P. 56230, Chapingo, México. E-mail: espinoal@inifap2.inifap.conacyt.mx y espinoale@yahoo.com.mx

4 Colaboradores de la Cátedra "Semillas”, Ingeniería Agrícola, FESC-UNAM, Cuautitlán Izcalli, México. 
Cuautitlán - Universidad Nacional Autónoma de México (UNAM), se han realizado diversos trabajos con la finalidad de incorporar la esterilidad masculina, a las líneas de los híbridos comerciales desarrollados en la Universidad, con fuentes diversificadas del carácter, así como el manejo racional del esquema. Desde 1992 se realizan trabajos para incorporar el carácter de androesterilidad a las líneas básicas de la UNAM, así como definir las líneas con capacidad restauradora. En los años 1995 a 1997 se evaluaron combinaciones de líneas con progenitores androesteriles para tratar de detectar genotipos con capacidad restauradora, encontrándose y verificándose que siete líneas restauran la fertilidad masculina. En los dos ciclos señalados se evaluaron 32 híbridos experimentales simples, obtenidos del cruzamiento de líneas androestériles con líneas posibles restauradoras de la fertilidad, de estos materiales 9 resultaron fértiles en un $100 \%$ y 3 con fertilidad parcial, lo que indica que las líneas EHT-29-2, EHT-49-3, IA49-1, IA49-2 y P2-1 son restauradoras de la fertilidad y que los genes de la restauración están en condición homocigótica dominante, en cambio las líneas 43-1, EHT-30-5 y EHT-9-6 tienen los genes restauradores en forma heterocigótica, y el resto de las líneas paternas tienen los genes de la restauración en condición homocigótica recesiva. Las líneas restauradoras de la fertilidad en un $100 \%$ generaron híbridos con buenas características agronómicas y las no restauradoras generaron híbridos, que por sus excelentes características agronómicas, se pueden utilizar para formar híbridos trilineales con alguna de las líneas que se identificaron como restauradoras de la fertilidad. El uso de la androesterilidad y la capacidad restauradora de la fertilidad masculina en la producción de semilla híbrida de maíz, limita los problemas que ocurren en la eliminación de la panoja en progenitores hembra, reduciendo las dificultades en esta etapa facilitando el control de la calidad e identidad genética de los híbridos producidos además de una reducción en los costos de producción por labor de despanojado de 25 hasta 50 jornales por hectárea.

La androestérilidad dejó de utilizarse durante en la década de los 70's, debido a la susceptibilidad a la enfermedad del tizón foliar causada por el hongo $\mathrm{Hel}$ minthosporium maydis raza $\mathrm{T}$, que ocasionó una epifitia en gran parte de la faja maicera en E.U., con el descubrimiento de nuevas fuentes de esterilidad masculina, esta práctica se ha retomado con las nuevas fuentes, para no depender de una sola y limitar de esta manera los problemas generados con la raza T. Por otra parte en los Valles Altos las condiciones agro climáticas podrían limitar el desarrollo del hongo responsable del tizón foliar. En este trabajo se plantearon como objetivos: Identificar líneas de maíz de Valles Altos con capacidad restauradora de la fertilidad masculina a través de su progenie, Verificar la capacidad restauradora de la fertilidad masculina de las líneas de maíz identificadas, en híbridos androestériles de Valles Altos. Así como evaluar el rendimiento y las características agronómicas de estos híbridos.

\section{MATERIALES Y MÉTODOS}

En 1992 se iniciaron los trabajos para incorporar la androesterilidad a las líneas endogámicas de maíz que se manejan en la UNAM, estas actividades se efectuaron en las parcelas de la Facultad de Estudios Superiores Cuautitlán, ubicada dentro del municipio de Cuautitlán Izcalli, Estado de México a una altitud de $2252 \mathrm{msnm}$. La temperatura media anual es de $15,7^{\circ} \mathrm{C}$, el mes más frío es enero con $11,8{ }^{\circ} \mathrm{C}$ en promedio, con $2,3{ }^{\circ} \mathrm{C}$ de temperatura mínima y máxima de $26,5^{\circ} \mathrm{C}$. La precipitación media anual es de $605 \mathrm{~mm}$. Después de contarse con las líneas en su versión de esterilidad, además de verificarse que las fuentes fueran estables en localidades y condiciones climáticas, los genotipos androesteriles con tres y cuatro retrocruzas hacia la líneas que fueron convertidas a esterilidad masculina, se manejaron experimentos en el ciclo P-V de 1995, en donde se sembraron 32 híbridos simples obtenidos a través del cruzamiento de líneas androestériles con líneas que se consideraba que podrían poseer capacidad restauradora de la fertilidad masculina, las cuales también fueron sembradas en lotes contiguos. Cuando los híbridos llegaron a floración se efectuaron diariamente revisiones para verificar si los órganos masculinos, producían anteras y a su vez estas liberaban polen, con lo cual se identificó su capacidad restauradora de la fertilidad masculina, las líneas restauradoras se cruzaron con los híbridos androestériles, nuevamente y estas combinaciones fueron evaluadas en 1996, en donde se verificó la presencia o no de la fertilidad masculina de los 41 híbridos que contenían la combinación de androesterilidad por una línea restauradora, identificada así el ciclo anterior; además se evaluó su rendimiento comparándolos con nueve híbridos fértiles experimentales y dos híbridos comerciales como testigos. Las variables evaluadas fueron: floración masculina y femenina, porcentaje de plantas fértiles y estériles (relación del número total de plantas por parcela y el número de plantas fértiles).

\section{RESULTADOS Y DISCUSIÓN}

Los resultados obtenidos por los restauradores indican que se clasificaron en las siguientes opciones: 1) Cuando todas las plantas de la parcela son fértiles, tie- 
nen genes de restauración en condición homocigótica. 2) Si todas las plantas son estériles quiere decir que la línea es homocigótica recesiva. 3) Cuando la mitad de las plantas es fértil y la otra mitad es estéril, quiere decir que la línea fértil usada es heterocigótica.

Los híbridos (CxP4PL7)X P7-1 y (CxP10PL4)X P7-1, resultaron $100 \%$ estériles, por lo que la línea macho P7-1 es no restauradora de la fertilidad masculina. El híbrido (CxP4PL7)X P7-1 supera al (CxP10PL4)X P7-1 en características agronómicas y en rendimiento, siendo dos días más precoz el híbrido (CxP4PL7)X P71. Otro de los híbridos que resultaron androestériles es el híbrido (CxP7PL7)X 242-4, por lo que la línea 2424 no posee capacidad restauradora de la fertilidad masculina y expresó un rendimiento bueno con 9384 kg/ha. (Cuadro 1). La línea EHS-3-6 no es restauradora de la fertilidad masculina debido a que el híbrido (CxP4PL7)X EHS-3-6 resultó androestéril. Otra línea no restauradora de la fertilidad masculina fue la 242-3, que es progenitor para los híbridos (CxP4PL7) X242-3 y (CXP4PL7)X 242-3.

La línea EHT-9-6, la cual fue progenitora para los híbridos (CxP4PL7) X EHT-9-6 y (CxP10PL4) X EHT9-6, produjo híbridos parcialmente estériles en un 20 y $48 \%$ respectivamente. El híbrido (CxP4PL7) X EHT-96 fue de los más precoces de los híbridos evaluados, con 73 días a floración masculina y 76 días a floración femenina. Otra línea parcialmente restauradora de la fertilidad masculina fue la EHT-30-5, la misma que es progenitora del híbrido (CXP5PL3) X EHT-30-5, el

Cuadro 1. Resultados del ciclo P-V 1995 para las variables porcentaje de plantas fértiles y estériles, número de días a floración masculina y femenina y rendimiento en el ensayo de androesterilidad en híbridos de maíz para Valles Altos. Cuautitlán, México.

\begin{tabular}{|c|c|c|c|c|c|}
\hline \multirow{2}{*}{ Genealogía } & \multirow{2}{*}{$\begin{array}{c}\text { Rendimiento } \\
\text { (kg/ha) }\end{array}$} & \multicolumn{2}{|c|}{ Porcentaje de plantas } & \multicolumn{2}{|c|}{ Días a floración } \\
\hline & & Fértiles & Estéril & Masc. & Fem. \\
\hline (CxP7PL7) EHT-1-5 & 14119 & 0 & 100 & 77 & 80 \\
\hline (CxP7PL7) P2-1 & 13383 & 100 & 0 & 82 & 83 \\
\hline (CxP10PL4) P2-1 & 13256 & 100 & 0 & 77 & 85 \\
\hline (CxP7PL4) EHT-29-2 & 13186 & 0 & 100 & 83 & 83 \\
\hline (CxP7PL7) EHT-29-2 & 12160 & 0 & 100 & 82 & 83 \\
\hline (CxP10PL4) 43-1 & 12604 & 0 & 100 & 77 & 82 \\
\hline (CxP10PL4) 43-1 & 10513 & 0 & 100 & 80 & 80 \\
\hline (CxP4PL7) EHT-21-4 & 12337 & 0 & 100 & 80 & 85 \\
\hline (CxP4PL7) EHT-21-4 & 8684 & 0 & 100 & 80 & 83 \\
\hline (CxP4PL7) 241-4 & 12295 & 0 & 100 & 75 & 80 \\
\hline (CxP5PL3) 241-4 & 10224 & 0 & 100 & 80 & 82 \\
\hline (CxP7PL7) EHT-11-4 & 12236 & 0 & 100 & 82 & 83 \\
\hline (CxP3PL3) EHT-50-8 & 12100 & 0 & 100 & 75 & 82 \\
\hline (CxP4PL7) EHT-9-6 & 12061 & 80 & 20 & 73 & 76 \\
\hline (CxP10PL4) EHT-9-6 & 11231 & 52 & 48 & 73 & 76 \\
\hline (CxP10PL4) 43-3 & 11541 & 0 & 100 & 80 & 83 \\
\hline (CxP5PL3) IA49-2 & 11499 & 100 & 0 & 91 & 95 \\
\hline (CxP4PL7) IA49-2 & 7810 & 100 & 0 & 89 & 94 \\
\hline (CxP3PL3) 64-2 & 11479 & 0 & 100 & 82 & 82 \\
\hline (CxP4PL7) P7-1 & 11029 & 0 & 100 & 77 & 80 \\
\hline (CxP10PL4) P7-1 & 10452 & 0 & 100 & 77 & 82 \\
\hline (CxP3PL3) EHT-29-1 & 10844 & 100 & 0 & 77 & 83 \\
\hline (CxP4PL7) IA49-1 & 10704 & 100 & 0 & 89 & 95 \\
\hline (CxP5PL3) 243-3 & 10273 & 0 & 100 & 80 & 82 \\
\hline (CxP5PL3) EHT-30-5 & 9789 & 52 & 48 & 76 & 82 \\
\hline (CxP7PL7) 242-4 & 9384 & 0 & 100 & 76 & 82 \\
\hline (CxP4PL7) EHS-3-6 & 9242 & 0 & 100 & 73 & 80 \\
\hline (CxP4PL7) EHT-49-3 & 8985 & 100 & 0 & 80 & 85 \\
\hline (CxP7PL4) EHT-49-3 & 6802 & 100 & 0 & 80 & 83 \\
\hline (CxP4PL7) 242-3 & 8975 & 0 & 100 & 73 & 80 \\
\hline (CxP4PL7) 242-3 & 8099 & 0 & 100 & 77 & 80 \\
\hline (CxP10PL4) 42-5 & 8688 & 0 & 100 & 75 & 80 \\
\hline
\end{tabular}


cual tuvo un 52\% de fertilidad, un rendimiento de 9789 $\mathrm{kg} / \mathrm{ha}, 82$ días a floración femenina y 76 días a masculina, la altura de planta fue de $235 \mathrm{~cm}$ y la de mazorca $136 \mathrm{~cm}$. Cuando los híbridos son $100 \%$ fértiles, como: (CxP7PL7) X P2-1 y (CxP10PL4) X P2-1, al tener como progenitor masculino a la línea $\mathrm{P} 2-1$, con capacidad restauradora de la fertilidad masculina. Para la variable rendimiento los híbridos (CxP7PL7) X P2-1 y (CxP10PL4) X P2-1 alcanzan los más altos valores $(13383 \mathrm{~kg} / \mathrm{ha}$ y $13256 \mathrm{~kg} / \mathrm{ha}$ respectivamente), superados tan solo por el híbrido androestéril (CxP7PL7) X EHT-1-5 que rindió $141199 \mathrm{~kg} / \mathrm{ha}$, esto es natural debido a que, parte de la energía destinada a producir polen en híbridos androestériles se va hacia la producción de grano. Es importante resaltar que éstos híbridos superan numéricamente en rendimiento a 20 híbridos androestériles (Cuadro 1).

La línea IA49-2 generó los híbridos (CxP5PL3) x IA49-2 y (CxP4PL7) x IA49-2, 100\% fértiles, lo que indica que posee capacidad restauradora de la fertilidad masculina, esto reviste gran importancia debido a que es progenitora del híbrido PUMA 1157, el cual fue liberado junto con Puma 1159 y compite con los maíces comerciales de la zona de transición (1800-2200 msnm) de diversas empresas semilleras (Solano et al. 1996).

El híbrido (CXP3PL3) X EHT-29-1 resultó 100\% fértil, por lo que su línea progenitora EHT-29-1 es restauradora de la fertilidad masculina, La línea IA49-1, hermana de la IA49-2, como se esperaba también es restauradora de la fertilidad masculina, ya que el híbrido (CxP4PL7) x IA49-1 para el cual es progenitor masculino resultó $100 \%$ fértil y con un rendimiento de $10704 \mathrm{~kg} / \mathrm{ha}$. Los resultados señalados se confirmaron con la evaluación hecha en 1996, donde los materiales con fertilidad restaurada volvieron a mostrar esta característica (Cuadro 2). De esta manera, las líneas P2-1 y EHT-49-3, que en el ciclo anterior se habían identificado como restauradoras de la fertilidad, confirmaron su capacidad restauradora, ya que generaron híbridos $100 \%$ fértiles en todos los casos en que participaron como progenitores masculinos, además presentan los más altos rendimientos, como el híbrido UHS95E0123 X P2-1, el cual superó a todos los híbridos evaluados, presentando un rendimiento de $12230 \mathrm{~kg} / \mathrm{ha}$, lo cual indica que además de ser restauradora de la fertilidad masculina, genera híbridos con excelentes rendimientos (Phoelman 1990, Jugenheimer 1990). La línea EHT-493 , también generó híbridos con excelente rendimientos.

En general los híbridos evaluados fueron precoces (característica muy deseable en Valles Altos) y presentan sincronía a floración. El testigo comercial H-135 resulta ser el más tardío, por presentar el mayor número
Cuadro 2. Condición de fertilidad y comparación de medias (Tukey 0,05 de probabilidad) de rendimiento, floración masculina y femenina, del ensayo de androesterilidad en Valles Altos, Primavera-verano, 1996. Cuautitlán, México.

\begin{tabular}{|c|c|c|}
\hline Híbrido & $\begin{array}{l}\text { Rendimiento } \\
(\mathrm{kg} / \mathrm{ha})\end{array}$ & $\begin{array}{l}\text { Condición de } \\
\text { fertilidad }\end{array}$ \\
\hline UHS95E0123 X P2-1 & $12230 \mathrm{a}$ & Fértil \\
\hline UHS95E0131 X EHT-49-3 & $11645 \mathrm{ab}$ & Fértil \\
\hline UHS95E0108 X EHT-49-3 & $11593 \mathrm{ab}$ & Fértil \\
\hline UHS95E0115 X EHT-49-3 & $11354 \mathrm{abc}$ & Fértil \\
\hline UHS95E0126 X EHT-49-3 & $10521 \mathrm{abcd}$ & Fértil \\
\hline UHS95E0132 X EHT-49-3 & $10380 \mathrm{abcd}$ & Fértil \\
\hline UHS95E0107 X EHT-49-3 & $10283 \mathrm{abcd}$ & Fértil \\
\hline UHS95E0111 X EHT-49-3 & $10143 \mathrm{abcd}$ & Fértil \\
\hline UHS96E0247 (TESTIGO) & 10116 abcd & Fértil \\
\hline UHS95E0101 X EHT-49-3 & 10049 abcd & Fértil \\
\hline UHS95E0115 X EHT-11-1 & 9914 abcd & Parcialmente \\
\hline UHS95E0109 X EHT-11-1 & 9872 abcd & Estéril \\
\hline PUMA 1157 (TESTIGO) & 9863 abcd & Fértil \\
\hline UHS95E0129 X EHT-49-3 & $9861 \mathrm{abcd}$ & Fértil \\
\hline UHS95E0115 X EHT-9-6 & 9717 abcd & Fértil \\
\hline UHS95E0113 X EHT-11-1 & 9545 abcd & Fértil \\
\hline UHS95E0123 X EHT-49-3 & 9539 abcd & Fértil \\
\hline UHS95E0108 X EHT-11-1 & 9445 abcd & Estéril \\
\hline UHT96E0246 (TESTIGO) & 9321 abcd & Fértil \\
\hline UHS95E0119 X EHT-49-3 & 9177 abcde & Fértil \\
\hline UHS95E0101 X EHT-11-1 & 9057 abcde & Parcialmente \\
\hline UHS95E0105 X EHT-49-3 & 9057 abcde & Fértil \\
\hline UHS95E0104 X EHT-9-6 & 9 055abcde & Parcialmente \\
\hline UHS95E0122 X EHT-49-3 & 9032 abcde & Fértil \\
\hline UHS95E0104 X EHT-11-1 & 9009 abcde & Estéril \\
\hline UHS95E0101 X P2-1 & 8931 abcde & Fértil \\
\hline UHS95E0110 X EHT-11-1 & 8785 abcde & Estéril \\
\hline UHS95E0118 X EHT-11-1 & 8731 abcde & Fértil \\
\hline UHS95E0117 X EHT-49-3 & 8672 abcde & Fértil \\
\hline UHS95E0108 X EHT-30-5 & 8639 abcde & Fértil \\
\hline UHS95E0125 X EHT-9-6 & 8612 abcde & Fértil \\
\hline UHT96E0242 (TESTIGO) & 8510 abcde & Fértil \\
\hline UHT96E0245 (TESTIGO) & 8449 abcde & Fértil \\
\hline UHS95E0107 X EHT-9-6 & 8332 abcde & Fértil \\
\hline UHS95E0119 X EHT-9-6 & 8244 abcde & Parcialmente \\
\hline UHS95E0109 X EHT-30-5 & 8200 abcde & Fértil \\
\hline UHS95E0110 X EHT-49-3 & 8199 abcde & Fértil \\
\hline UHT96E0244 (TESTIGO) & 8 190abcde & Fértil \\
\hline UHS95E0130 X EHT-30-5 & 8149 abcde & Fértil \\
\hline UHS95E0130 X EHT-49-3 & 8131 abcde & Fértil \\
\hline UHS95E0131 X EHT-30-5 & 8094 abcde & Parcialmente \\
\hline UHT96E0243 (TESTIGO) & 8029 bcde & Fértil \\
\hline UHS95E0125 X EHT-49-3 & 7650 bcde & Fértil \\
\hline UHS95E0132 X EHT-30-5 & 7644 bcde & Parcialmente \\
\hline UHS95E0111 X EHT-30-5 & 7532 bcde & Fértil \\
\hline H-135 (TESTIGO) & 7498 bcde & Fértil \\
\hline UHS95E0130 X EHT-9-6 & 7287 cde & Fértil \\
\hline UHS96E0248 (TESTIGO) & 6580 de & Fértil \\
\hline UHS95E0101 X EHT-30-5 & $6501 \mathrm{de}$ & Fértil \\
\hline UHS95E0117 X EHT-30-5 & 5019 e & Fértil \\
\hline DHS & 4149 & \\
\hline
\end{tabular}


de días a floración masculina con 89 días y femenina 94 días, lo cual lo hace estadísticamente diferente a todos los híbridos evaluados.

Los híbridos que le siguen en precocidad al H-135 son el testigo comercial PUMA 1157, con 84 días a floración masculina y 85 días a floración femenina, compartiendo valores iguales con el testigo experimental UHT96E0242 en floración femenina y con el testigo experimental UHT96E0245 en floración masculina, a su vez estos híbridos son superados en precocidad por 43 híbridos experimentales que se encuentran distribuidos entre los 74 a 82 días a floración masculina y los 75 y 82 días a floración femenina.

\section{CONCLUSIONES}

Se encontraron varias líneas restauradoras de la fertilidad, como P2-1, IA49-2, IA49-1, EHT-29-1 y EHT-49-3, las cuales contienen los genes de la restauración en estado homocigótico, ya que generaron híbridos 100\% fértiles. Las líneas EHT-11-1, EHT-30-5 y EHT-9-6, tienen capacidad que restaura la fertilidad masculina, sin embargo ésta probablemente está en situación heterocigótica, ya que generaron híbridos parcialmente fértiles. Los híbridos que resultaron androésteriles, y que presentaron buenas características agronómicas, podrían ser utilizados para formar híbridos triliniales con las líneas que se identificaron con capacidad restauradora de la fertilidad masculina. Las líneas EHT-49-3 y P2-1, confirmaron su capacidad restauradora de la fertilidad masculina, infiriéndose que se encuentran en condición homocigótica. Además estás líneas generan híbridos con buen potencial agronómico, por los altos rendimientos alcanzados de 8 a 12 ton/ha que superan numéricamente a los testigos comerciales PUMA 1157 y H-135. Las líneas EHT-11-1, EHT-30-5 y EHT-9-6, confirmaron su capacidad restauradora de la fertilidad masculina al generar híbridos parcialmente fértiles, lo cual podría significar que posee esta capacidad de restauración, pero en estado heterocigótico, por lo que presentan segregación, y deben ser utilizadas bajo un esquema especial, sin embargo debido a que producen híbridos con buen potencial agronómico deben ser aprovechadas. Las líneas IA49-
2, IA49-1, EHT-49-3 y P2-1, al mostrar en ambos ciclos la capacidad restauradora de la fertilidad masculina al $100 \%$, resultan de importancia, ya que no requieren de un esquema especial de producción para ser utilizadas como restauradoras, además algunas de estas líneas fueron progenitoras de híbridos con buenas características agronómicas dentro de los cuales se incluye al PUMA 1157. El uso de líneas con la característica esterilidad masculina así como otras con capacidad restauradora, son una buena alternativa para la producción de semilla híbrida de maíz, se incrementan los rendimientos, se disminuyen los costos y lo más importante se mantiene la calidad genética de los híbridos

\section{LITERATURA CITADA}

AIRY, J. M.; TATUM, L. A.; SORENSON, J. W. 1978. La producción de semillas, producción de semilla híbrida de maíz y sorgo para grano. In: Anuario Estadístico de Semillas. Traducido por Antonio Marino y Pánfilo Rodríguez. Editorial C.E.C.S.A. México. pp. 274-285.

CURTIS, D. L. 1983. Algunos aspectos de la producción de semilla de Zea mays L. (Maíz) en E.U.A. In: Producción Moderna de Semilla. Tomo II. (Trad. Federico Stanham). Ed. Agropecuaria Hemisferio Sur S. R. L. Uruguay. pp. 467-479.

FEISTRITZER, W.P.; BRADLEY, R.; OGADA, F. 1983. Producción y recolección de semillas. In: Colección FAO. Tecnología de la Semilla de Cereales. FAO. Italia. p.63.

JUGENHEIMER, R. W. 1990. Maíz, variedades mejoradas, métodos de cultivo y producción de semilla. Noriega Limusa. México. pp. 150, 489-502, 591-601.

PÉREZ, A. R. 1964. Restauración de la fertilidad por maíces mexicanos en la fuente $\mathrm{T}$ de esterilidad citoplasmática masculina. Tesis de Maestría. Colegio de Posgraduados Chapingo. México pp. 5-7

PHOELMAN, J. M. 1987. Mejoramiento genético de las cosechas. Limusa. México. pp. 278-280.

SÁNCHEZ E., A. 1988. Producción de semillas de maíz híbrido. In: Producción y manejo de semillas. SARH. Tamaulipas-Norte. pp. 57-61. 